\title{
ONICOMICOSIS EN PACIENTES CON INFECCIÓN POR VIH (TUCUMAN, ARGENTINA)
}

\author{
(Onychomycosis in patient with VIH infections (Tucumán, Argentina)
}

\author{
Raquel Salim ${ }^{1}$ \& Rosa Runco ${ }^{1,2}$ \\ 1. Cátedra de Micología. Instituto de Microbiología "Dr. Luis C. Verna". \\ Facultad de Bioquímica, Química y Farmacia - Universidad Nacional de Tucumán. \\ Ayacucho 491. (4000) San Miguel de Tucumán. Argentina. \\ 2. Laboratorio de Micología del Hospital del Niño Jesús. Pasaje Hungría 750. \\ (4000) San Miguel de Tucumán. Argentina. \\ e-mail: rqsalim@unt.edu.ar
}

Palabras clave: Onicomicosis, pacientes VIH seropositivos, diagnóstico micológico, mohos.

Key words: Onychomycosis, VIH seropositive patient, mycological diagnostic, moulds.

\section{RESUMEN}

En el presente estudio se evalua la etiología y prevalencia de onicopatías micóticas en pacientes VIH seropositivos para un mejor conocimiento de su incidencia e importancia clínica.

Para determinar su incidencia se estudiaron 140 muestras clínicas provenientes de 102 pacientes VIH seropositivos que incluyeron 9 niños y 93 adultos de ambos sexos, de 2 a 50 años de edad, con manifestaciones clínicas compatibles con onicomicosis en manos, pies o ambas localizaciones. Mediante examen directo, cultivos, estudios micromorfológicos y bioquímicos, se confirmó la etiología micótica en 68 pacientes (66,6\%). El 57,3\% de los cultivos correspondieron a Candida spp.; el 25,6\% a dermatofitos y el resto $(17,1 \%)$ a mohos no dermatofitos. En total 46 (32,8\%) muestras clínicas arrojaron resultados negativos. El mayor número de casos perteneció al grupo de edades entre 31 y 40 años (38,2\%), sexo masculino (55,8 \%), con localización en pie $(50,0 \%)$ y menos de tres años de evolución.

Aún cuando más del $50 \%$ de las enfermedades que atacaron las uñas fueron causadas por hongos, un porcentaje importante de onicopatías fueron de etiología no micótica (32,8\%), lo que reafirma que diversas enfermedades en las uñas tienen expresión morfológica similar

Consideramos que el diagnóstico diferencial constituye un sólido argumento para darle importancia al estudio micológico en la evaluación de cualquier patología ungueal.
ABSTRACT

The present study was made to evaluate the etiology and the predominance of onychomycosis in HIV seropositive patients and to improve the knowledge on their incidence and clinical significance.

To determine their incidence 140 clinical samples collected from $102 \mathrm{HIV}$ seropositive patients (9 children and 93 adults, male and female, 2 to 50 years old) with clinical signs compatible with onychomycosis in hands, feet or both, were studied.

The mycotic etiology in 68 patients (66.6\%) coul be confirmed by means of direct exam, cultures as well as micromorphological an biochemical studies. The 57.3\% of the positive cultures corresponded to Candida spp.; $25.6 \%$ to dermatophytes and $17.1 \%$ to nondermatophyte molds. As a total 46 (32,8\%) clinical samples were negative. Thirty one and forty year old patients (38.2\%),males (55.8\%),bearing feet infection (50,5\%) and with a lower than 3 year evolution period revealed the highest incidence.

Even though more than $50 \%$ of diseases affecting nails were caused by fungi, a significant percentage of onychomycosis had a non mycotic etiology $(32.8 \%)$ proving that different lesions occurring in nails develop a similar morphological pattern.

We think that a differential diagnostic represent a solid argument in order to stress the importance of the mycological studies to evaluate any onychomycosis pathology. 


\section{INTRODUCCIÓN}

La onicomicosis, una infección fúngica de la uña, puede ser causada por varias especies de dermatofitos, levaduras y mohos no pertenecientes a los dermatofitos. Estas afecciones, que alteran la función normal de las uñas, pueden causar considerable dolor, interfieren con las actividades cotidianas y producen efectos psicosociales negativos que pueden interferir con la vida personal y profesional del individuo (4).

Las onicomicosis pueden confundirse con muchas onicopatías: psoriasis, liquen plano, paroniquia crónica, dermatitis crónica, leuconiquias parciales y adquiridas de origen traumático, onicodistrofias traumáticas, onicomadesis, alteraciones congénitas, onicolisis idiopática, enfermedades hormonales, enfermedad de Reiter, etc. El diagnóstico diferencial constituye un sólido argumento para darle importancia al estudio micológico en la evaluación de cualquier patología ungueal ya que la instauración de un tratamiento antifúngico no justificado de una onicomicosis falsamente diagnosticada, puede ser causa de un trastorno considerable no exento de contraindicaciones y de peligros potenciales.

Las diferentes formas clínicas de infección fúngica en uñas están bien documentadas $(6,13,27)$. Se describen cuatro tipos principales de onicomicosis de acuerdo a la presentación clínica y la ruta de invasión fúngica: 1) subungueal distal y lateral; 2) proximal subungueal; 3) blanca superficial y 4) onicomicosis por Candida. Cada uno de estos cuatro tipos de onicomicosis tiene distintos patógenos causales y tanto la forma de invasión fúngica en la placa ungueal como la presentación clínica tienen características distintas. La progresión clínica de la infección de cualquiera de ellas puede llevar a la forma distrófica total con la destrucción de la lámina ungueal hasta el nivel de la lúnula.

La onicomicosis subungueal distal es la más común, afecta la porción distal o lateral del lecho ungueal y la cara interna de la uña. Las condiciones clínicas asociadas son: hiperqueratosis subungueal, onicolisis y paroniquia. El principal agente patógeno responsable es T. rubrum, también pueden causarla T. mentagrophytes y $\boldsymbol{E}$. floccosum.

En la onicomicosis proximal subungueal el hongo penetra a través de la cutícula e invade la porción proximal del lecho subungueal a la altura de la matriz de la uña y se extiende en sentido distal como una mancha blanca opaca situada profundamente. Aparece más frecuentemente en las uñas de los pies que de las manos (26). La presentación clínica puede incluir hiperqueratosis subungueal, onicolisis proximal, leuconiquia y destrucción de la tabla ungueal proximal (6). Es causada primariamente por $\boldsymbol{T}$. rubrum pero también por $\boldsymbol{T}$. menta- grophytes y T. tonsurans. Este tipo de lesión es la forma clínica menos común en individuos sanos $(5,7)$, sin embargo, es considerada un marcador clínico temprano de infección VIH y se observa frecuentemente en estos pacientes $(3,7,8,12,22,24)$.

La onicomicosis blanca superficial es el tipo menos común y se estima en aproximadamente el $10 \%$ de los casos (5). Aparece como una mancha blanca superficial sobre la porción dorsal de la tabla ungueal, principalmente del ortejo mayor. Mientras la lesión progresa la uña se vuelve rugosa, blanda y se desmenuza. El agente etiológico más común es $\boldsymbol{T}$. mentagrophytes pero se han encontrado varios hongos no dermatofitos causando esta micosis, entre ellos Aspergillus terreus, Acremonium roseogriseum, Acremonium potronii y Fusarium oxysporum $(14,25,28)$.

El cuarto tipo, onicomicosis por Candida, es frecuentemente causada por $\boldsymbol{C}$. albicans y presenta tres subtipos: a) paroniquia, la forma más común de las 3 , es marcada por inflamación y eritema de los pliegues proximal y laterales de la uña; b) onicolisis, la tabla ungueal se separa del lecho subungueal y c) el granuloma que se caracteriza por invasión directa y engrosamiento de la uña asociada a paroniquia. Este tipo de infección ocurre principalmente en los pacientes inmunocomprometidos (23).

Las micosis en uñas son probablemente las enfermedades más comunes en los adultos, con una prevalencia estimada del 8,7\% (26). Su incidencia aumenta y está en expansión en poblaciones cuyos factores de riesgo son: diabetes, inmunosupresión (trasplantes, VIH, terapias inmunosupresoras, cobaltoterapia o quimioterapia y terapéutica prolongada con corticosteroides o antibióticos), psoriasis, hiperhidrosis, y traumatismos ungueales. En personas mayores de 70 años de edad la incidencia aumenta a más del $40 \%$. Esta aumentada susceptibilidad, puede deberse a la reducción de la función inmune relacionada a la edad y adicionalmente a la reducida eficiencia vascular en esta población $(12,13)$.

Varios estudios informan que aproximadamente el 92\% de los pacientes con infección por VIH presentan manifestaciones dermatológicas extensas de la piel o las uñas y que la gama de agentes etiológicos se amplía al sumarse patógenos oportunistas a los patógenos tradicionales, con producción de cuadros clínicos atípicos, más severos, recidivantes que dificultan el diagnóstico y que resultan menos sensibles a los tratamientos convencionales. La posibilidad de tratamiento de una onicomicosis en el paciente seropositivo constituye un auténtico desafío (16).

$\mathrm{Al}$ revisar la literatura encontramos pocos estudios relacionados con el tema en nuestro país, por lo que efectuamos el presente trabajo con la finalidad de eva- 
luar la etiología y prevalencia de onicopatías micóticas en pacientes VIH seropositivos en Tucumán, Argentina, para mejorar el conocimiento sobre la incidencia e importancia clínica de este tipo de infecciones en nuestro medio.

\section{MATERIALES Y METODOS}

Se procesaron 140 muestras de uñas procedentes de 102 pacientes VIH seropositivos.

En la confección de la ficha de datos del paciente se registraron edad, sexo, localización, tiempo de evolución, forma y aspecto de la lesión (subungueal distal, subungueal proximal, blanca superficial, onicolisis, paroniquia, lesión distrófica total) y porcentaje de uñas afectadas.

Las muestras se obtuvieron por raspado con bisturí estéril de la tabla ungueal y el espacio subungueal (lecho y cara interna) de uñas con signos de onicomicosis. La muestra se obtuvo raspando además el surco periungueal en los casos de lesiones en pliegues proximal y/o laterales. Las escamas fueron recogidas entre dos portaobjetos esterilizados por flameado.

Se realizaron exámenes micológicos directos por digestión alcalina en caliente con KOH 20-30\% entre porta y cubreobjetos. Los cultivos, en medio de agar Sabouraud-glucosado, adicionado de penicilina (50 U.I./ $\mathrm{ml}$ ) y estreptomicina (80 ug/ml), fueron incubados a $28^{\circ} \mathrm{C}$ y $37^{\circ} \mathrm{C}$ durante 15 a 20 días.

Los dermatofitos fueron identificados siguiendo la metodología de Rebell y Taplin (21) que incluye estudios macro y micromorfológicos de los cultivos, hidrólisis de urea y producción de órganos perforadores del pelo in vitro.

Para la identificación de las levaduras aisladas se emplearon las pruebas convencionales de formación de tubos germinativos, producción de clamidoconidios, desarrollo en CHROMagar-Candida (medio cromogénico para el aislamiento y diferenciación de especies de Candida), Candi-Fast para zimogramas y API-20 (BioMerieux) para auxanogramas.

Los mohos no dermatofitos oportunistas se identificaron por sus características macro y micromorfológicas de acuerdo a de Hoog y Guarro (2). Estos hongos fueron considerados agentes causales cuando fueron aislados en cultivo puro y abundantes colonias o en dos muestras consecutivas del mismo paciente.

\section{RESULTADOS}

En el lapso 1998-2001 se estudiaron 140 muestras de uñas provenientes de 102 pacientes VIH seropositivos, 9 niños y 93 adultos, de 2 a 50 años de edad, de ambos sexos, con manifestaciones clínicas compatibles con onicomicosis en manos, pies o ambas localizaciones, con una evolución mayor al año. La composición de la población estudiada fue: 55,8\% varones y $44,2 \%$ mujeres. El 7,8\% eran menores de 12 años.

En 94 muestras clínicas $(67,2 \%)$ se aislaron hongos, confirmándose onicopatía de etiología micótica en 68 pacientes (66,6\%). El porcentaje de resultados negativos totales fue del 32,8\%.

El grupo etario de mayor incidencia fue el de $31-40$ años (38,2\%) seguido del de $41-50$ años (23,5\%) con predominio en el sexo masculino (Figura 1).

En su mayoría los pacientes presentaban varias uñas afectadas, el 50,0\% con lesiones sólo en uñas de pies, el 38,2\% con lesiones simultáneas en manos y pies, y el resto $(11,8 \%)$ con localización sólo en manos (Figura 2). Las lesiones en las uñas del ortejo mayor fueron las más frecuentes. Cinco pacientes (7,4\%) presentaron sus 20 uñas afectadas.

El 57,3\% de los cultivos correspondieron a especies de Candida, el 25,6\% a dermatofitos y el resto $(17,1 \%)$ a mohos no dermatofitos (Tabla 1$)$.

El orden de frecuencia de las especies aisladas fue: C. tropicalis 25,4\%; C. albicans 21,3\%; C. parapsilosis 6,4\%; C. krusei 2,1\%; C. guilliermondii 2,1\%; T. rubrum 17,0\%; T.mentagrophytes 4,3\%; $\boldsymbol{E}$. floccosum 4,3\%; Scopulariopsis brevicaulis 4,3\%; Fusarium oxysporum 4,3 \%; Aspergillus niger 4,3\%; Aspergillus terreus 2,1\%; y Acremonium spp. 2,1\%.

Se observó una franca dominancia de levaduras en uñas de manos, mientras que los dermatofitos predominaron en los pies. Los agentes etiológicos aislados de uñas de las manos fueron: $\boldsymbol{C}$. albicans, $\boldsymbol{C}$. tropicalis y $\boldsymbol{C}$. guilliermondii. En uñas de los pies se observó un franco predominio de $\boldsymbol{T}$. rubrum sobre $\boldsymbol{T}$. mentagrophytes y $\boldsymbol{E}$. floccosum.

En el 100\% del grupo de 0-12 años de edad se aisló $\boldsymbol{C}$. tropicalis tanto en uñas de las manos como de los pies.

Los pacientes con lesiones simultáneas en manos y pies presentaron tres situaciones diferentes: I) se aisló el mismo agente causal en ambas localizaciones en el 61,5\% de los casos; II) se diagnosticó onixis por Candida en manos y tinea unguium en los pies en el 30,8\% de los pacientes y III) en dos pacientes (7,7\%) se encontró C. tropicalis en manos y Acremonium spp. en pie.

Los mohos no dermatofitos asentaron solamente en uñas de los pies.

El $66,1 \%$ de los pacientes con onicomicosis presentaban la forma proximal subungueal. Los mohos no dermatofitos causaron también la forma blanca superficial, siempre con localización en uñas del pie, y prácticamente indistinguibles de las producidas por derma- 
Tabla 1. Localización de las lesiones y distribución de los agentes etiológicos

\begin{tabular}{|c|c|c|c|c|c|}
\hline \multicolumn{2}{|c|}{ AGENTES ETIOLOGICOS } & \multirow{2}{*}{$\begin{array}{c}\text { Manos } \\
10\end{array}$} & \multirow{2}{*}{$\frac{\text { Pies }}{14}$} & \multirow{2}{*}{$\begin{array}{c}\begin{array}{c}\text { Total de } \\
\text { Muestras } \\
(+)\end{array} \\
24\end{array}$} & \multirow{2}{*}{$\begin{array}{c}\% \\
25,4\end{array}$} \\
\hline & C. tropicalis & & & & \\
\hline & C. albicans & 14 & 6 & 20 & 21,3 \\
\hline \multirow[t]{4}{*}{ Candida $(57,3 \%)$} & C.parapsilosis & 2 & 4 & 6 & 6,4 \\
\hline & C. krusei & 0 & 2 & 2 & 2,1 \\
\hline & C. guilliermondii & 2 & 0 & 2 & 2,1 \\
\hline & T. rubrum & 2 & 14 & 16 & 17,0 \\
\hline \multirow[t]{4}{*}{ Dermatofitos $(25,6 \%)$} & T. mentagrophytes & 2 & 2 & 4 & 4,3 \\
\hline & E. floccosum & 2 & 2 & 4 & 4,3 \\
\hline & S. brevicaulis & 0 & 4 & 4 & 4,3 \\
\hline & F.oxysporum & 0 & 4 & 4 & 4,3 \\
\hline \multirow[t]{3}{*}{$\begin{array}{l}\text { Mohos no } \\
\text { dermatofitos }(17,1 \%)\end{array}$} & Aspergillus niger & 0 & 4 & 4 & 4,3 \\
\hline & Aspergillus terreus & 0 & 2 & 2 & 2,1 \\
\hline & Acremonium spp. & 0 & 2 & 2 & 2,1 \\
\hline TOTAL & & 34 & 60 & 94 & 100,0 \\
\hline
\end{tabular}

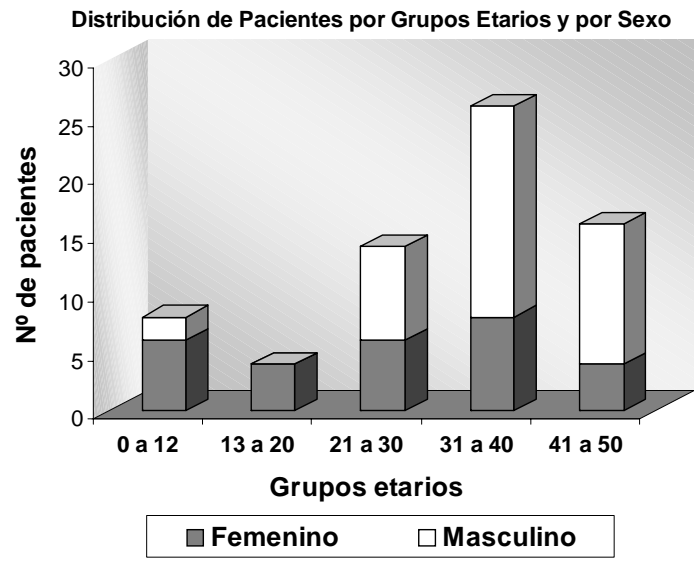

Figura 1.- Distribución de pacientes por grupos etarios y por sexo

tofitos. Seis pacientes presentaron melanoniquia en las uñas de ambos pies, aislándose $\boldsymbol{C}$. albicans en dos casos, C. tropicalis en tres y T. rubrum en uno.

\section{DISCUSION}

La pandemia del VIH, afecta a adultos y niños de todos los continentes. El impacto de la infección por VIH ha determinado un incremento estadísticamente sig-

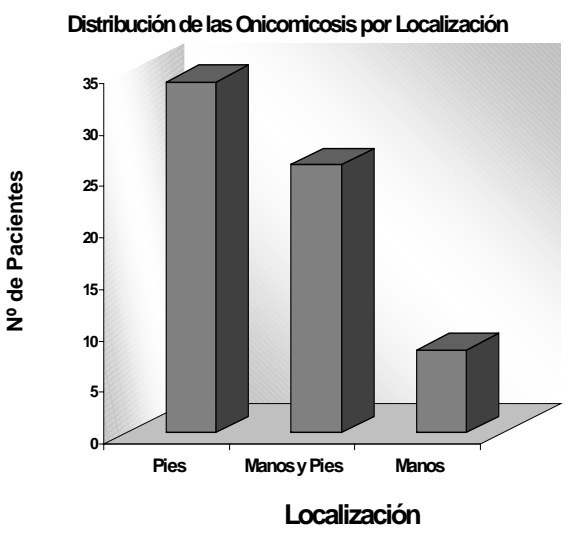

Figura 2.- Distribución de la onicomicosis por localización

nificativo de las infecciones fúngicas en general que pueden representar un 30\% del total de éstas (17). En los últimos años las micosis ungueales no sólo han visto aumentada su incidencia en forma notable sino que, además, hay una mayor diversificación de los agentes etiológicos (19).

Las personas con infección por VIH no sólo presentan una susceptibilidad aumentada a las onicomicosis, sino que sus infecciones tienden a ser más extensas y más 
refractarias al tratamiento y con una mayor tasa de recurrencia que la población general $(12,15)$.

La disparidad de los resultados sobre la incidencia de los diferentes agentes etiológicos en los pacientes infectados por el VIH es manifiesta y varía según los autores. Levy (13), en un estudio epidemiológico de onicomicosis en poblaciones de riesgo encontró entre un 11\% y $67 \%$ de pacientes con VIH afectados. En algunos países, el agente etiológico más importante es $\boldsymbol{T}$. rubrum $(3,8,12)$ y en menor medida $\boldsymbol{E}$. floccosum (11) mientras que en otros, la presencia de especies de Candida alcanza el 63\% (19,20). En general los diferentes autores señalan que las infecciones más comunes son las causadas por los dermatofitos, cuya incidencia es cuatro veces mayor que en la población general y que las infecciones más frecuentes son las de la piel o las onicomicosis $(5,12,18)$. Midgley (18), afirma que las onicomicosis en el paciente inmunodeficiente a menudo se presentan con variaciones tanto en su manifestación clínica como en las especies fúngicas involucradas. Con todo, los agentes etiológicos responsables son muy variados e incluyen a otros hongos oportunistas.

Un estudio realizado en el Hospital F. J. Muñiz de la ciudad de Buenos Aires, Argentina, incluyó a 86 pacientes con dermatoficias asociadas a enfermedad avanzada por VIH. Las onicomicosis se detectaron en más del $40 \%$ de los casos, en el $29,7 \%$ fueron exclusivamente del tipo proximal profundo y las restantes se asociaron a la presencia de onicomicosis distales subungueales, blancas superficiales y onicolisis en otras uñas (11).

El presente estudio, realizado entre 1998 y 2001 en nuestro medio, indica que las onicomicosis por Candida afectan al 57,3\% de los pacientes VIH seropositivos, con una mayor prevalencia de estas levaduras frente a los hongos dermatofitos (25,6\%). Contrariamente a los hallazgos de otros autores $\boldsymbol{C}$. albicans no fue el agente etiológico predominante $(10,19)$, sino que $\boldsymbol{C}$. tropicalis fue la especie más frecuentemente aislada $(25,4 \%)$, seguida por $\boldsymbol{C}$. albicans (21,4\%). La frecuencia con que aparecen las otras especies es variable y mucho menor. Por otro lado, las onixis candidósicas se presentaron con mayor frecuencia en las uñas de manos en mujeres mientras que en general en las uñas de pies en los hombres.

T. rubrum fue el dermatofito predominante y el responsable del 66,6\% de tinea unguium. Estos resultados coinciden con otros estudios realizados en pacientes con infección por VIH que consideran a T. rubrum el agente etiológico más frecuente e importante en uñas del pie $(3,5,12)$.

En el paciente con VIH, la infección micótica tiende a ser más severa, persistente y se disemina más rápidamente a otras uñas, de manos o pies $(13,15)$. Hemos observado 5 casos de leuconiquia de las 20 uñas, lo que en nuestro conocimiento sólo fue previamente descrito por Cribier et.al., en 1998 (1).

Las onicomicosis producidas por hongos filamentosos no dermatofitos son clínicamente similares a las causadas por dermatofitos. Su incidencia e importancia no es bien conocida ya que pueden ser colonizadores o contaminantes más que patógenos. Coincidentemente con nuestros resultados, los mohos causantes de onicomicosis citados más frecuentemente son Aspergillus, Acremonium, Fusarium y Scytalidium siendo Scopulariopsis brevicaulis la especie más común (6, 9, 14, 25).

Estudios hechos en otros países revelan que el $12 \%-15 \%$ de micosis ungueales fueron causados por estos agentes oportunistas, presentando examen directo y repetidos cultivos positivos. Los autores consideran que este número de aislamientos no es despreciable y que su frecuencia está en aumento (18). Por otra parte hay coincidencia entre diferentes investigadores en cuyos resultados destacan que las formas clínicas más frecuentes fueron la onicomicosis blanca superficial y subungueal distal. Los mismos autores señalan que las uñas de los pies son las que se infectan más que las de las manos en ambos sexos $(9,18)$ lo que corrobora nuestros hallazgos.

En nuestro estudio hemos aislado el 17,1\% de cultivos positivos de mohos no dermatofitos, casi siempre limitados al ortejo mayor, siendo ligeramente más alta la incidencia en mujeres. No hemos encontrado compromiso de las uñas de las manos por estos agentes. Consideramos que en estos casos, conocer el estado inmunológico del paciente es clave al momento de la valoración etiológica del hallazgo de estos agentes causales. Los datos epidemiológicos y etiológicos están influenciados por la patología, factores cronológicos, el área geográfica, la existencia de factores predisponentes y de patogenicidad. Al existir variaciones geográficas entre las distintas tasas de aislamiento es difícil extraer conclusiones generales sobre la etiología y epidemiología que permitan unificar en una sola tabla los datos de incidencia de los distintos agentes etiológicos en todo el mundo. Es evidente la necesidad de disponer de datos epidemiológicos y etiológicos con el fin de mejorar el conocimiento sobre la incidencia e importancia clínica de estas patologías y para elegir el tratamiento más adecuado. Esta exigencia es aún mayor en aquellas patologías como las que nos ocupan, en las que son necesarios tratamientos difíciles y para las que se han descrito una mayor cantidad de fracasos terapéuticos y de recidivas.

Los procedimientos usados para determinar la presencia de hongos en el material de uñas es simple y aún cuando se requiere experiencia y habilidad para reconocer las características microscópicas de los agentes causales, no requieren ninguna infraestructura sofisticada. 
Sin embargo, para la distinción entre las infecciones producidas por dermatofitos y la identificación de otros hongos no dermatofitos, se requiere de una experiencia adicional en micología.

Si bien en este estudio más del $50 \%$ de las enfermedades que atacan la uña son causadas por hongos, hemos encontrado un porcentaje importante de onicopatías de etiología no micótica $(32,8 \%)$ lo que confirma que en las uñas diversas enfermedades tienen expresión morfológica similar, lo que pone de relieve la necesidad del entrenamiento en el reconocimiento de formas clínicas atípicas y de la confirmación micológica del laboratorio para su diagnóstico definitivo y terapéutica oportuna y eficaz.

Consideramos que el diagnóstico micológico diferencial en pacientes con infección por VIH, es importante en la evaluación de cualquier patología ungueal, donde la producción de formas clínicas atípicas, severas y recurrentes, dificultan el diagnóstico y complican el tratamiento.

\section{REFERENCIAS}

1. Cribier, B. (1998). Nail changes in patients infected with Human Immunodeficiency Virus. Arch. Dermatol. 134:1216-1220

2. De Hoog G.S. \& Guarro, J. (1995). Atlas of Clinical fungi, Central Bureau voor Scmmelcultures/Universitat Rovira i Virgili, Baarn and Delft. The Netherlands/ Reus, Spain.

3. Dompmartin. D., A. Dompmartin et al. (1990). Onychomycosis and AIDS: clinical and laboratory findings in 62 patients. Int. J. Dermatol. 29:337-339.

4. Drake, L.A.; Sherr, R.K. \& Smith, E.B. (1998). Efecct of onychomycosis on quality of life. J.Am Acad. Dermatol. 38:702-704

5. Elewsky, B.E. (1998). Onychomycosis in special-risk populations. Clin. Microbiol.

Rev. 11:415-428

6. Elewsky, B.E.; Rinaldi, M.G. \& Weitzman I. (1995). Diagnosis and Treatment on onychomycosis: a clinician's handbook. Calif. N.J. GardinerCaldwell Synermed; 5:13-24

7. Elewsky,B.E., (1993). Clinical pearl: proximal white subungual onychomycosis in AIDS. J.Am.Acad.Dermatol 29:631-632

8. Elewsky,B.E \& Hay, R.J. (1996). Update on the management of onychomycosis: highlights of the Third Annual International Summit on Cuatneous Antifungal Therapy. Clin. Infectr. Dis 23:305-313

9. García-Martos, P.; Domínguez, I. \& Marín, P. (2000). Onicomicosis por hongos filamentosos no dermatofitos en Cádiz. Enf. Infecc. Microbiol Clín. 18:319-324

10. Hazeb, K. (1995). New and emerging yeast pathogens. Clin. Microb. Rev. 5:462-478

11. http://caibco.ucv/vitae/VitaeNueve.htm/ Micosis Asociadas al SIDA. Parte $2^{\mathrm{a}}$. Dermatoficias.

12. Jaffe,R. (1998). Onychomycosis: recognition, diagnosis and management. Arch.Fam.Med. 7:587-592

13. Levy, L.A. (1997). Epidemiology of onychgomycosis in special-risk populations. J. Am. Podiatr..Med. Assoc. 87:546-550

14. López-Jodra, O. \& Torres-Rodríguez, J.M. (1999). Especies fúngicas poco comunes responsables de onicomicosis. Rev. Iberoam.Mnicol 16:S11-S15
15.Loveland L. (1998). Onychomycosis in HIV-positive patients. Clin. Podiatr. Med. Surg. 15:305-315

16. Martínez-Rojano, H. \& Morales Quintero, C. (2000). Manifestaciones mucocutáneas en niños nacidos de madres VIH-seropositivas. Rev. Am. Pedr. 67:214-219

17. Midgley,G.; Moore, M. \& Cook., J. (1994). Mycology of nail disorders. J. Am. Acad. Dermatol 31:68-74

18. Midgley ,G. \& Moore, M. (1998). Onychomycosis. Rev. Iberoam. Micol. 15:113-117

19. Pereiro, M.; Losada-Campa A. \& Iglesias, A. (1994). Estudio comparativo de la flora levaduriforme en pacientes VIH+ y un grupo control. Rev. Iberoamer Micol 11:S-36

20. Perfect, J.R. \& Schell, W.A. (1996). The new fungal opportunists are coming. Clin Infect. Dis. 22:112-118

21. Rebell ,G. \& Taplin, D. (1979) Dermatophytes: their recognition and identification, Coral Gables, Fla, University of Miami Press.

22. Rongioletti, F. A.; Persi., S.; Tripodi, \& Rebora A..(1994). Proximal white subungueal onychomycosis: a fign of immunodeficiency. J.Am.Acad.Dermatol 30:129-130

23. Scher,R.; Odom, R. \& Elewsky, B.E. (1995). Superficial fungal infections: emphasis on chronic and recurrent cases, onychomycosis, and the HIV patient. Clin.Courier 14:11

24. Silva-Lizana, E. (1996). Proximal white subungual onychomycosis in AIDS. Int. J. Dermatol. 35:290-291

25. Torres-Rodríguez, J.M. \& López-Jodra, O. (1999). Etiologic agents of onychomycosis from toe nails. Multicentric Spanish study. J. Eur. Acad. Dermatol. Veneorol.11:25-31

26. Trepanier, E.F., \& Amsdem, G.W. (1998). Current issues in onychomycosis. Ann Pharmacother. 32:204-214

27. Zaias, N. (1992). Clinical manifestations of onychomycosis. Clin. Exop. Dermatol. 17:S6-S7

28. Summerbell, R. C., J. Kane, and S. Krajden. 1989. Onychomycosis, tinea pedis, and tinea manuum caused by non-dermatophytic filamentous fungi. Mycoses 32:609-619. 